\title{
Restrictions in eligibility for long-acting reversible contraception (LARC) -- what other viable options are available for birth control and family planning?
}

Keywords: contraception, birth control, family planning

\section{Opinion}

The paper critically assesses recent publications addressing prominent topics in family and community medicine, such as the efficacy of contraceptive methods and in particular long-acting reversible contraceptives (LARCs). Its aim is to discuss the problem of limited eligibility to LARC and to draw attention to the question of safety. In examining contraceptive methods that guarantee both efficacy and safety the paper analyses international studies and concludes by suggesting that new research on efficacy replace obsolete data hitherto used in the scientific literature on family and community medicine. Given the importance of contraception for socio-economic as well as public-health reasons, it is not surprising that virtually all leading medical journals regularly publish articles on birth control and family planning. A most recent publication focuses exclusively on long-acting reversible contraceptives (LARCs), which are described as the "most highly effective"/sic!) methods. ${ }^{1}$ One of the most noteworthy contributions of this study is the discussion of eligibility. As the table for "Medical Eligibility Criteria (MEC) for initiation of LARC Methods" ( p.464) shows, quite a number of women are not eligible for LARCs due to conditions such as current pelvic inflammatory disease, persistent intrauterine gestational trophoblastic disease, unexplained vaginal bleeding, etc. It is, however, not sufficient to consider such restrictions; equally important is the phenomenon of intolerance towards hormones and aversion to drugs or devices. In addition a considerable number of women refrain from using LARCs for socio-economic reasons, because of a socio-cultural background, or due to religious convictions. The question therefore arises as to whether alternatives could be offered to women in the $38 \%$ segment of U.S. non-users of contraception. One alternative has been propounded implicitly as early as 2011, when research on contraceptive technology ${ }^{2}$ presented assessments together with a contraceptive failure table. According to this table estimates for some methods, that do not use hormons or devices, approximate those of some LARCs. For example, the 0.4 perfect use estimate for the symptothermal method compares favorably with the 0.6 estimate for ParaGard (copper T), an intrauterine device (IUD).

This fertility awareness-based method which is free from side effects and risks would therefore be a viable option, provided the users are capable of absolute adherence to the requirements of the method, which means measuring basal body temperature, examining cervical mucus, and observing general symptoms such as mastalgia of the peri-ovulatory cycle phase. As regards safety, LARCs are supassed also by three other no-risk methods with acceptable perfect use estimates namely Ovulation (3\%), TwoDay (4\%), and Standard Days (5\%), according to the Contraceptive Technology Failure Table. ${ }^{2}$ Given the importance of safety of contraceptive methods for many women -- which means in this context side effect and risks but not protection against sexually transmitted diseases -- it should be borne in mind that already in 1999, a widely-used international reference
Volume 2 Issue 2 - 2018

\author{
Kurt Kraetschmer \\ Austrian American Medical Research Institute, Austria
}

Correspondence: Kurt Kraetschmer,Austrian American Medical Research Institute, Austria, Email kurt.kraetschmer@aon.at

Received: October 20, 2017 | Published: March 21, 2018

book described the symptothermal method as the most precise among the periodic abstinence methods, whose safety has remained uncontested. ${ }^{3}$ German research has highlighted the method in 2000 as a generally reliable method and the most reliable among the natural family planning methods, due to a Pearl index of 0.8 for perfect use. ${ }^{4}$ A prospective longitudinal study of 2007 has furnished additional data on the efficacy of the method. ${ }^{5}$ Presently, organisations and institutions such as the American Congress of Obstetricians and Gynecologists (ACOG),${ }^{6}$ the Guttmacher Institute, ${ }^{7}$ the University of Wisconsin, ${ }^{8}$ or the Mayo Clinic ${ }^{9}$ attribute failure rates to the method which are comparable to those of some of the 19 methods included in the U.S. Food and Drug Administration survey of contraceptive methods, eg, cervical cap with spermicide or sponge with spermicide. ${ }^{10}$ In contrast to the incomplete information provided by the U.S. FDA -- where symptothermal, ovulation, TwoDays and Standard Days are not mentioned -- the ACOG draws attention to advantages of the so-called "fertility awareness methods," by stating: "They cost very little. . . Many women like the fact that fertility awareness is a form of birth control that does not involve the use of medications or devices. ${ }^{\text {" }}$ The additional advantage with respect to achieving pregnancy is underscored by the U.S. Office of Population Affairs which considers the "sympto-thermal" method as a combination of Basal Body Temperature (BBT) and cervical mucus. ${ }^{11}$ In light of such findings, there is reason to believe that not only the $45 \%$ of U.S. unintended pregnancies but also the $34 \%$ of Western Europe could still be reduced. ${ }^{1}$ By disseminating accurate information on both efficacy and safety through public health media and during counselling sessions in the doctor's office, women who are not eligible for LARCs or avoid them for other reasons could nevertheless be motivated to engage in efficacious birth control and family planning so that the U.S. $38 \%$ segment of non-users of contraception could be reduced.

\section{Acknowledgement}

None.

\section{Conflict of interest}

The author declares no conflict of interest. 


\section{References}

1. Curtis KM, Peipert J. Long Acting Reversible Contraception. $N$ Engl J Med. 2017;376(5):461-468.

2. Trussell J. Contraceptive efficacy, Table 3-2. Hatcher RA, Trussell J, editors. Contraceptive Technology: Twentieth Revised Edition. New York, NY: Ardent Media; 2011.

3. Beers MH, Berkow R, MSD Manual. Whitehouse Station, $17^{\text {th }}$ ed, N.J. USA: Merck \& Co; 1999.

4. Gröger S, Grüne B. Kontrazeption. et al. Gynäkologie und Geburtshilfe. Berlin, Springer; 2000:60-87.
5. Frank-Herrmann P, Heil J, Gnoth C, et al. The effectiveness of a fertility awareness-based method to avoid pregnancy in relation to a couple's sexual behaviour during the fertile time: a prospective longitudinal study. Human Reproduction. 2007;22(5):1310-1319.

6. American Congress of Obstetricians and Gynecologists FAQ; USA; 2017.

7. Guttmacher Institute; New York; 2016.

8. University of Wisconsin; USA; 2017.

9. Mayo Clinic.

10. U.S.Food and Drug Administration. USA; 2017.

11. Office of Population Affairs; 2017. 